\title{
A NEW FORMULATION OF THE THEORY OF STRONG COUPLING
}

\author{
Th. W. RUIJGROK \\ Institute for Theoretical Physics, \\ Rijksuniversiteit, Utrecht, The Netherlands
}

Received 19 July 1968

\begin{abstract}
Using the scalar symmetrical theory with a fixed and extended source as a model, we show how, in a simple way, the Hamiltonian can be written as $H=H_{\mathrm{O}}+g V$, where $V$ is independent of the coupling constant $g$. The term $H_{\mathrm{o}}$, which does depend on $g$, can easily be diagonalized. We demonstrate that the effect of $g V$ on the energy spectrum is small for large as well as for small $g$. The Schrödinger equation takes a form which allows not only the determination of extra terms in the asymptotic expansion of the energy, but also a fast and accurate numerical calculation for all values of the coupling constant. This makes it possible to compare and evaluate the one- and two-point Padé and other approximations.
\end{abstract}

\section{INTRODUCTION}

The recent [1] interest and success in the theory of strong interactions is mainly due to the desire to generate baryon spectra. As in the older literature [2] the underlying idea is always to consider the meson-nucleon interaction as the main part of the Hamiltonian and to take the kinetic energy of the meson field as the perturbation. Complications arise because the mes. on-nucleon interaction not only causes nucleon isobars to exist, but should also explain the meson-nucleon scattering. In order to eliminate this difficul. ty we will consider only mesons with a given energy (equal to the rest mass) so that only the discrete part of the spectrum remains. In sect. 2 we will show how, for this reduced system, the Schrödinger equation can be written in a rather simple form. This form is tentamount to a new splitting of the Hamiltonian [as can be seen from eqs. (5.1), (5.2) and (5.3)] in which the main part of the original perturbation, but not all, together with the meson field energy now forms the new unperturbed Hamiltonian. The remaining perturbation has a small effect both for small and for large coupling constants. The numerical calculation of the energy of the ground state for a given total isotopic spin can be performed very quickly and is exhibited in sect. 3 for a large range of coupling constants. In sect. 4 these exact results are compared with a perturbation calculation (small $g$ ) and with a Padé approximation derived from it. In the same section we also give an 
approximation of the ground state energy, which is valid for large values of the isotopic spin. It is shown that in this case the series expansion in powers of $g^{2}$ has a radius of convergence which tends to zero. The strong coupling limit, together with a two-point Padé approximation is discussed in sect. 5. A number of intermediary mathematical results is collected in an appendix.

\section{A NEW FORM OF THE EIGENVALUE PROBLEM}

The system we want to describe consists of one fixed nucleon interacting with a charged scalar meson field. The interaction must be invariant for rotations in isospin space. In order to reduce the mathematical difficulties to a minimum we make the additional assumption that only s-wave mesons with a definite energy (equal to the rest mass $\mu=1$ ) interact with the nucleon. The Hamiltonian for this system is

$$
H=D+g \sum_{l=1}^{3} C_{l} \tau_{l},
$$

where

$$
D=\sum_{l=1}^{3} a_{l}^{*} a_{l}
$$

is the energy of the free mesons, $C_{l}=a_{l}+a_{l}^{*}$, $\tau_{l}$ are the Pauli (iso)spin operators and $g$ is the coupling constant. For each half-integer value $j$ of the total isospin we will try to find the state of lowest energy and with third component of the isospin also equal to $j$. All other states with different $I_{3}$ can then be found by application of $I_{-}$. Excited states with the same total isospin will not be considered because in a realistic theory they would probably fall in the continuous part of the spectrum. The states we are looking for can be written in the form

$$
|j\rangle=\alpha A_{J-1}^{J-1}|\mathrm{p}\rangle+\frac{\beta}{\sqrt{2 J+1}}\left[\sqrt{2 J} A_{J}^{J}|\mathrm{n}\rangle-A_{J}^{J-1}|\mathrm{p}\rangle\right]
$$

where $J=j+\frac{1}{2}$ and $J-1=j-\frac{1}{2}$ are the two values of the isospin of the meson field which, together with a proton $|p\rangle$ or neutron $|n\rangle$, can combine to a state of total isospin $j$. We have introduced the tensor operators $A_{k} q$ which, when acting on the meson vacuum $|0\rangle$, create a normalized state of the meson field with total isospin $k$ and third component $q$. The weight factors $\alpha$ and $\beta$ are to be determined later, but such that $|\alpha|^{2}+|\beta|^{2}=1$. The problem is now reduced to finding the operators $A_{k} q$. Substituting eq. (2.2) into the equation $H|j\rangle=\epsilon|j\rangle$ and equating the coefficient of $|\mathrm{p}\rangle$ on the leftand the right-hand side gives 


$$
\begin{aligned}
{\left[\alpha D A_{J-1}^{J-1}-\frac{\beta}{\sqrt{2 J+1}} D A_{J}^{J-1}+\alpha g C_{O} A_{J-1}^{J-1}+\frac{\beta g}{\sqrt{2 J+1}} \sqrt{4 J} C_{+} A_{J}^{J}\right.} \\
\left.\quad-\frac{\beta g}{\sqrt{2 J+1}} C_{O} A_{J}^{J-1}\right]|0\rangle=\epsilon\left[\alpha A_{J-1}^{J-1}-\frac{\beta}{\sqrt{2 J+1}} A_{J}^{J-1}\right]|0\rangle .
\end{aligned}
$$

The coefficient of $|\mathrm{n}\rangle$ could be obtained by applying $I_{+}$to eq. (2.3) and therefore gives no extra information.

In order to expand the left hand side of eq. (2.3) in tensor operators we remark that $A_{k} q$ can always be written as

$$
A_{k}^{q}=T_{k}^{q} F \text {, }
$$

where $F$ is the most general scalar operator which can be formed from the meson creation operators and where $T_{k} q$ is defined by

$$
T_{k}^{k}=\frac{\left(a_{+}^{*}\right)^{k}}{\sqrt{k !}}
$$

and the commutator relations

$$
\begin{aligned}
& {\left[I_{3}, T_{k}^{q}\right]=q T_{k}^{q},} \\
& {\left[I_{+}, T_{k}^{q}\right]=\sqrt{(k-q)(k+q+1)} T_{k}^{q+1},} \\
& {\left[I_{-}, T_{k}^{q}\right]=\sqrt{(k+q)(k-q+1)} T_{k}^{q-1} .}
\end{aligned}
$$

By forming contractions with $\delta_{k l}$ and $\epsilon_{k l m}$ of products of the form $a_{k}^{*} a_{l}^{*} \ldots a_{m}^{*}$ one can form scalar operators. Since, however, all mesons have the same energy, all contractions formed with $\epsilon_{k l m}$ vanish. The most general scalar operator $F$ is therefore a function $F\left(G^{*}\right)$ of $G^{*} \equiv \sum_{j=1}^{3} a_{j}^{*} a_{j}^{*}$. We now write

$$
\begin{gathered}
A_{J-1}^{q}=T_{J-1}^{q} F_{1}\left(G^{*}\right), \quad A_{J}^{q}=T_{J}^{q} F_{2}\left(G^{*}\right), \\
F_{1}=\sum_{n=0}^{\infty} P_{n} G^{* n}, \quad F_{2}=\sum_{n=0}^{\infty} Q_{n} G^{* n},
\end{gathered}
$$

and substitute these expressions into eq. (2.3). Using the formulae (A.1)-(A.8) of the appendix the left-hand side can be written as a sum of terms of the form (2.4). The coefficient of $T_{J+1}^{J-1} G^{* n}|0\rangle$ is zero, whereas the coefficients of $T_{J-1}^{J-1} G^{* n}|0\rangle$ and of $T_{J}^{J-1} G^{* n}|0\rangle$ give rise to the following two equations

$$
\alpha(2 n+J-1) P_{n}+\frac{\beta g}{\sqrt{2 J+1}} Q_{n-1}+\frac{\beta g}{\sqrt{2 J+1}}(2 n+2 J+1) Q_{n}=\epsilon \alpha P_{n}
$$




$$
\frac{\beta}{\sqrt{2 J+1}}(2 n+J) Q_{n}+\alpha g P_{n}+\alpha g(2 n+2) P_{n+1}=\frac{\epsilon \beta}{\sqrt{2 J+1}} Q_{n},
$$

$\left(n=0,1,2, \ldots ; Q_{-1} \equiv 0\right)$.

Introducing

$$
\begin{aligned}
X_{2 n} & =\left[\frac{(J-1) ! n !(2 n+2 J-1) !}{(2 J-1) !(n+J-1) !}\right]^{\frac{1}{2}} P_{n}, \\
X_{2 n+1} & =\frac{\beta}{\alpha}\left[\frac{(J-1) ! n !(2 n+2 J+1) !}{2(2 J-1) !(2 J+1)(n+J) !}\right]^{\frac{1}{2}} Q_{n},
\end{aligned}
$$

the equations (2.5) take the more symmetrical form*

$$
\begin{aligned}
& (2 n+J-1) X_{2 n}+g\left\{\sqrt{2 n} X_{2 n-1}+\sqrt{2 n+2 J+1} X_{2 n+1}\right\}=\epsilon X_{2 n}, \\
& (2 n+J) X_{2 n+1}+g\left\{\sqrt{2 n+2 J+1} X_{2 n}+\sqrt{2 n+2} X_{2 n+2}\right\}=\epsilon X_{2 n+1},
\end{aligned}
$$

$(n=0,1,2, \ldots)$.

The normalization requirement

$$
\left\langle 0\left|\left(A_{k}^{q}\right)^{*} A_{k}^{q}\right| 0\right\rangle=1,
$$

for $k=J, J-1$ and $q=J, J-1$ gives for $X_{n}$ the conditions (using formula (A.9) of the appendix)

$$
\sum_{n=0}^{\infty}\left|X_{2 n}\right|^{2}=1 \quad \text { and } \quad \sum_{n=0}^{\infty}\left|X_{2 n+1}\right|^{2}=\left|\frac{\beta}{\alpha}\right|^{2}
$$

The eigenvalue spectrum is now completely determined by the boundary condition $\Sigma_{n=0}^{\infty}\left|X_{n}\right|^{2}<\infty$. Once the eigenvector $X_{n}$ has been found we obtain $|\beta / \alpha|$ from eq. (2.7).

\section{NUMERICAL SOLUTION}

The fact that we have a single boundary condition is a considerable improvement over the usual method where two functions, satisfying two coupled second order differential equations, must each satisfy certain conditions in the origin and at infinity. For large coupling constants the numerical solution is thereby made very cumbersome. In the present case the exact solution of eq. (2.6) for the ground state was calculated, using a $\mathrm{X}-8$ electronic computer. Using the facts that for the ground state $(-1)^{n} X_{n}$ has the same sign for all $n$ and that for $n$ larger than a certain value

* For $J=1$ this equation was obtained by Henley and Lee [6]. 


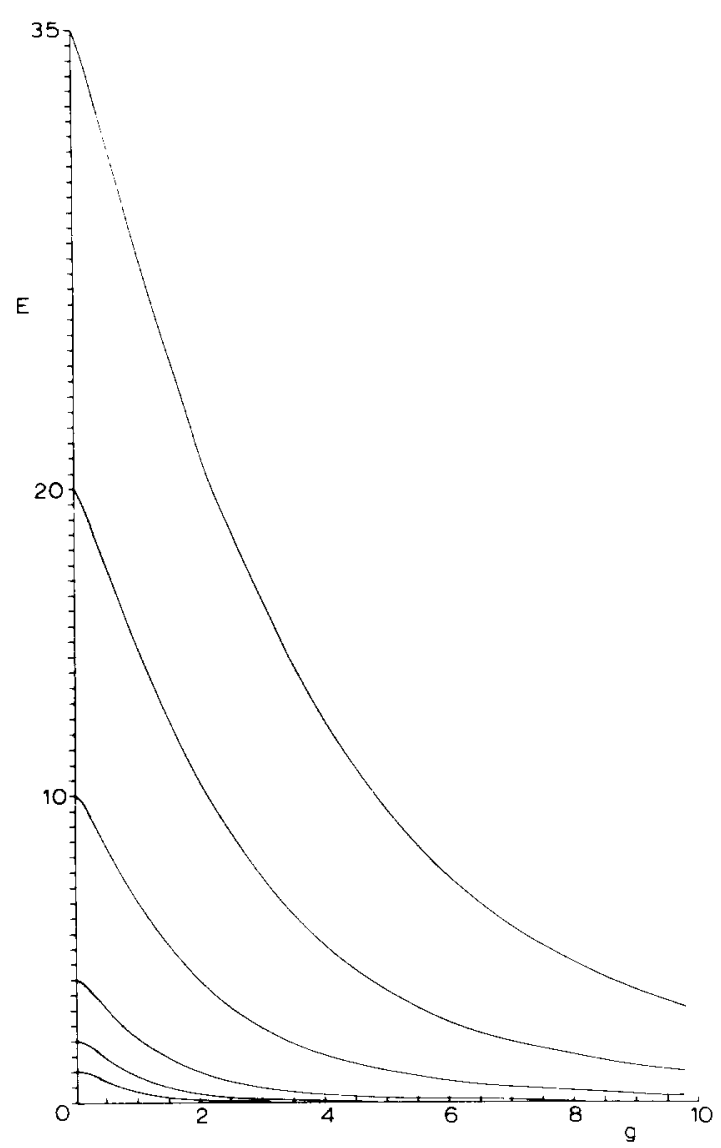

Fig. 1. Exact value of $E=\epsilon+g^{2}+1$ as a function of $g$ for different values of $J$.

$X_{n+1} / X_{n}<1$ a fast numerical method [3] was developed ${ }^{*}$, which for $J$ ranging between 1 and 40 and $g$ between 0 and 40 gave the value of $\epsilon$ with an absolute accuracy of $10^{-6}$.

In fig. 1 we have plotted $E \equiv \epsilon+g^{2}+1$ as a function of $g$ for different values of $J$. For $g$ going to zero this quantity approaches the value $J$, corre. sponding to the energy $J-1$ of the unperturbed ground state. The ordering of the levels which occurs at $g=0$ is maintained for all $g$-values, i.e. no level crossing occurs. We plot $E$, rather than $\epsilon$, because of the fact that $-g^{2}$ is the nucleon mass renormalization, which, in a theory where all momenta play a role, would be infinite.

In fig. 2 the function $|\beta(g)|^{2}$ is plotted for different $J$-values. From (2.2)

* I am very grateful to Mr.J.Renes for his invaluable help in performing the numerical calculations. 


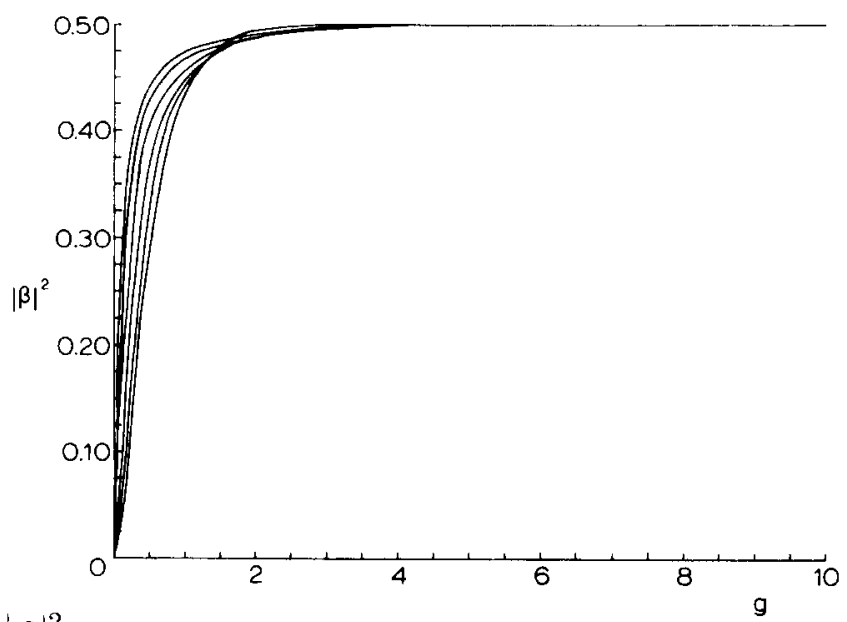

Fig. 2. $|\beta|^{2}$ as a function of $g$ for $J=1,2,3,10,20,40$. For $g=0.5$ the value of $|\beta|^{2}$ increases with increasing $J$.

it is seen that the meson field is in a mixed state and carries either isospin $J$ or isospin $J-1$. The function $|\beta|^{2}$ gives the relative weight of the isospin $J$ part. It is seen that with increasing $g$ and for all $J$ it very quickly approaches the value of fifty percent and is never larger than that. The renormalized coupling constant, defined as $g_{\mathrm{r}}=g\left\langle\frac{1}{2}\left|\tau_{3}\right| \frac{1}{2}\right\rangle$, was calculated and found to be equal to $g_{r}=\left(1-\frac{4}{3}|\beta|^{2}\right) g$. For $g \rightarrow \infty$ this leads to the known [5] result $g_{r} \rightarrow \frac{1}{3} g$.

In the following sections the limits of weak and strong coupling will be discussed and compared with the exact solutions of the present section.

\section{WEAK COUPLING}

For the ground state we try to find a power series solution for $X_{n}$ and $\epsilon$, i.e. we write

$$
\begin{aligned}
X_{0} & =1 \\
X_{2 n} & =\sum_{s=n}^{\infty} \frac{\sqrt{(2 J+1)(2 J+3) \ldots(2 J+2 n-1)}}{1.3 .5 \ldots(2 n-1) \sqrt{2.4 .6 \ldots 2 n}} A_{n s} g^{2 s},
\end{aligned}
$$

for $n=1,2, \ldots$,

$$
X_{2 n+1}=\sum_{s=n+1}^{\infty} \frac{\sqrt{(2 J+1)(2 J+3) \ldots(2 J+2 n+1)}}{1.3 .5 \ldots(2 n+1) \sqrt{2.4 .6 \ldots 2 n}} B_{n s} g^{2 s-1},
$$

for $n=0,1,2, \ldots$,

$$
\epsilon=\sum_{l=0}^{\infty} \epsilon_{l} g^{21}
$$


Substitution into eq. (2.6) gives the following equations for $A_{n s}$ and $B_{n s}$ :

$$
\begin{gathered}
2 n\left(A_{n s}+B_{n-1, s}\right)+\left(1+\frac{2 J}{2 n+1}\right) B_{n s}=\sum_{l=1}^{s-n} \epsilon_{l} A_{n, s-l}, \\
(2 n+1)\left(A_{n s}+B_{n, s+1}\right)+A_{n+1, s}=\sum_{l=1}^{s-n} \epsilon_{l} B_{n, s-l+1},
\end{gathered}
$$

where

$$
\epsilon_{0}=J-1 \text {, }
$$

has been used. For $s=n$, eqs. (4.1a) and (4.1b), respectively become

$$
2 n\left(A_{n n}+B_{n-1, n}\right)=0
$$

and

$$
(2 n+1)\left(A_{n n}+B_{n, n+1}\right)=0 .
$$

Since $A_{00}=1$ it follows that $A_{n n}=1$ and $B_{n, n+1}=-1$. From eq. (4.1a) follows with $n=0$ that

$$
\epsilon_{s}=(2 J+1) B_{0 s} .
$$

In particular $\epsilon_{1}=-(2 J+1)$. For the calculation of $B_{0 s}$ eqs. (4.1a) and (4.1b) must be applied alternatingly for the determination of the following series.

$$
\begin{gathered}
A_{s s}=1, B_{s-1, s+1}, A_{s-1, s+1}, B_{s-2, s+2}, A_{s-2, s+2}, \ldots, B_{0,2 s} \rightarrow \epsilon_{2 s}, \\
B_{s, s+1}=-1, A_{s, s+1}, B_{s-1, s+2}, A_{s-1, s+2}, \ldots, B_{0,2 s+1} \rightarrow \epsilon_{2 s+1} .
\end{gathered}
$$

In this way we calculated six terms in the series

$$
E=\epsilon+g^{2}+1=\sum_{s=0}^{\infty} E_{s} g^{2 s},
$$

and found

$$
\begin{aligned}
& E_{0}=J \\
& E_{1}=-2 J \\
& E_{2}=4 J^{2}+2 J \\
& E_{3}=-\left[16 J^{3}+10.666666 J^{2}+1.333333 J\right] \\
& E_{4}=80 J^{4}+67.555555 J^{3}+15.111111 J^{2}+0.666666 J, \\
& E_{5}=-\left[448 J^{5}+476.444444 J^{4}+165.540740 J^{3}+21.303703 J^{2}+0.822222 J\right] .
\end{aligned}
$$

This power series is a good approximation to $E$ only for $g^{2} \ll 1$.

Closed expressions for $E_{S}$ could not be obtained. It is, however, possible to find the leading term in $E_{S}$ and from that the behaviour of $E$ for large $J$. For that purpose we remark that after multiplication by $2 J+1$, formula $(4.1 \mathrm{~b})$ can for $n=0$ and $s \geqslant 1$ be written as 


$$
\epsilon_{S+1}=\epsilon_{1} \epsilon_{S}+\epsilon_{2} \epsilon_{S-1}+\ldots+\epsilon_{S} \epsilon_{1}-(2 J+1) A_{1 s} \cdot
$$

From (4.1) one sees that for large $J$ the behaviour of the different functions is given by

$$
A_{n s} \propto J^{s-n}, \quad B_{n s} \propto_{J} s-n-1, \quad \epsilon_{l} \propto J^{l} .
$$

In eq. (4.4) we can therefore neglect the last term in the right-hand side.

We now write the asymptotic form of $\epsilon$ as

$$
\tilde{\epsilon}\left(g^{2}\right)=\sum_{s=0}^{\infty} \tilde{\epsilon}_{s} g^{2 s},
$$

with $\tilde{\epsilon}_{0}=J-1$ and $\tilde{\epsilon}_{1}=-2 J$. Multiplying eq. (4.4) by $g^{2(s+1)}$ and summing over $s$ from 1 to infinity gives

$$
\begin{aligned}
\tilde{\epsilon}\left(g^{2}\right)-\tilde{\epsilon}_{0}-\tilde{\epsilon}_{1} g^{2}=\sum_{s=1}^{\infty} \tilde{\epsilon}_{s+1} g^{2 s+2} \\
\quad=\sum_{s=1}^{\infty} \sum_{\substack{m, n=1 \\
(m+n=s+1)}}^{\infty} \tilde{\epsilon}_{m} \tilde{\epsilon}_{n} g^{2(m+n)}=\left(\sum_{m=1}^{\infty} \tilde{\epsilon}_{m} g^{2 m}\right)^{2} \\
=\left[\tilde{\epsilon}\left(g^{2}\right)-\tilde{\epsilon}_{0}\right]^{2} .
\end{aligned}
$$

From this we obtain

$$
\widetilde{\epsilon}\left(g^{2}\right)=J-\frac{1}{2}-\frac{1}{2} \sqrt{1+8 g^{2} J} .
$$

The radius of convergence of the series (4.5) is clearly equal to $1 /(8 J)$. We may therefore conclude that the radius of convergence of the power series expansion (4.3) approaches zero for $J$ going to infinity. In order to see whether the expression (4.6) is still a good approximation to $\epsilon\left(g^{2}\right)$ for values of $g$ outside the radius of convergence we have plotted in fig. 3 the function $\tilde{\epsilon}\left(g^{2}\right)+g^{2}+1$ and the numerically calculated values of $\epsilon\left(g^{2}\right)+g^{2}+1$ for $J=1,2,4,10,20$ and 35. It is seen that (4.6) is a good approximation for $g^{2} \lesssim \frac{1}{4} J$, which is far outside the radius of convergence.

Eq. (4.6) indicates that $\epsilon\left(g^{2}\right)$ will have a branch line along the negative $g^{2}$-axis with a branch point approaching zero for increasing $J$.

In figs. 4 and 5 the Padé approximants $[1,1],[2,2],[2,1]$ and $[3,2]$ for $\epsilon+g^{2}+1$ are compared with the exact values. The $[2,1]$ and $[3,2]$ approximations show a very good agreement for all values of $J$, provided $g<0.6$. For larger values of $g$ higher $[N+1, N]$ approximations are required to describe the strong coupling region. This limit will be considered in the next section. 


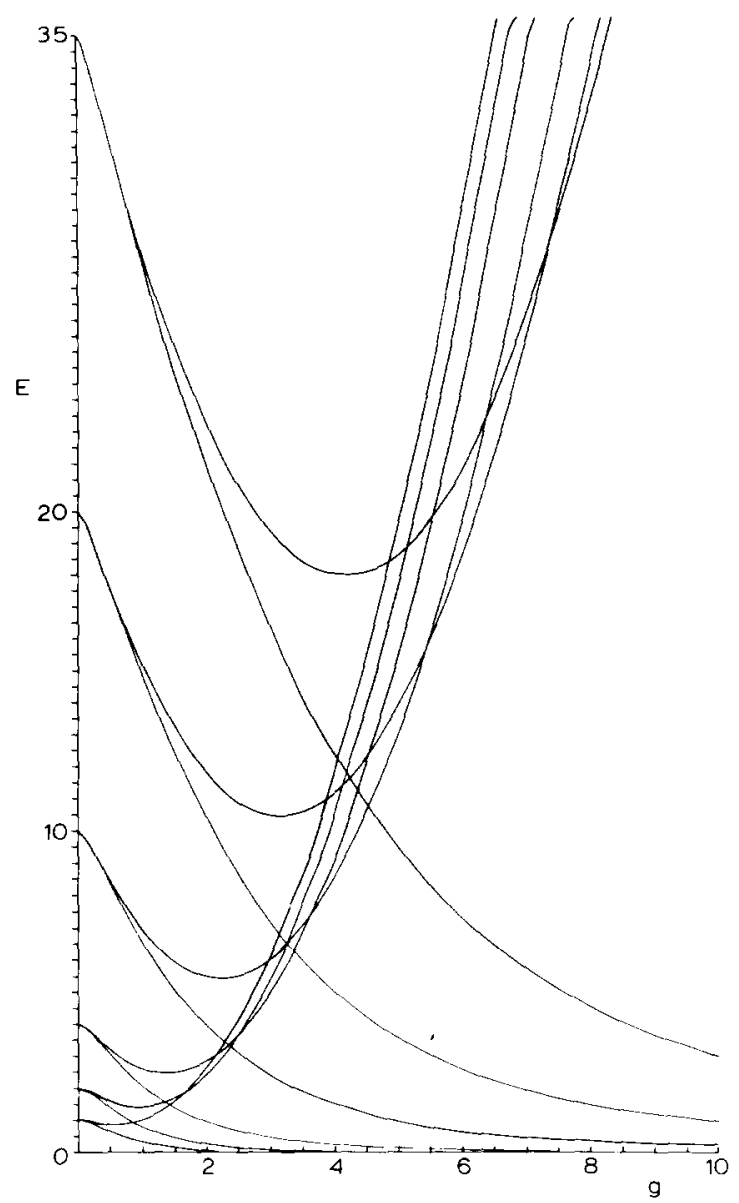

Fig. 3. Comparison of $\widetilde{\epsilon}+g^{2}+1$ [eq. (4.6)] with the exact value of $\epsilon+g^{2}+1$.

\section{STRONG COUPLING}

Eq. (2.6) can also be written in the form

$$
H \boldsymbol{X}=\epsilon \boldsymbol{X},
$$

where $H$ is the following hermitian matrix

$$
H=\left(\begin{array}{cccccc}
J-1 & g \sqrt{2 J+1} & & & & \\
g \sqrt{2 J+1} & J & g \sqrt{2} & & & \\
& g \sqrt{2} & J+1 & g \sqrt{2 J+3} & & \\
& & g \sqrt{2 J+3} & J+2 & g \sqrt{4} & \\
& & & g \sqrt{4} & J+3 & g \sqrt{2 J+5}
\end{array}\right)
$$




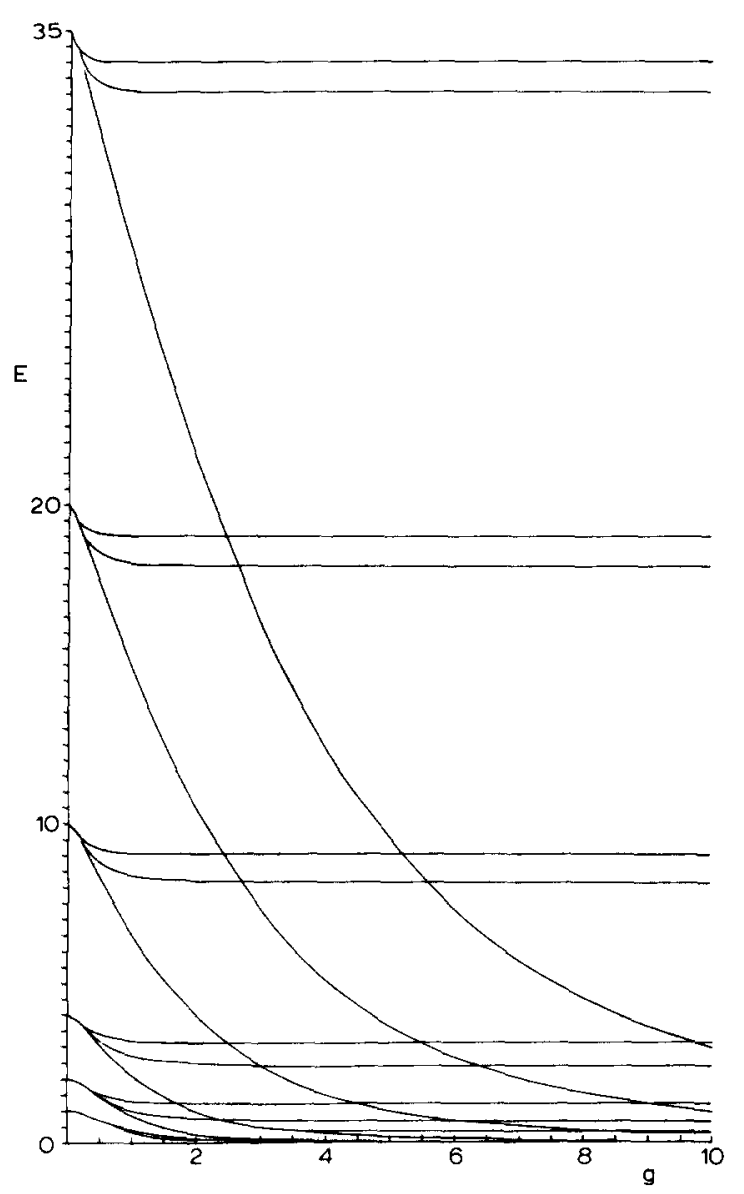

Fig. 4. Comparison of $[1,1]$ and $[2,2]$ Pade approximations with the exact value of $\epsilon+g^{2}+1$ for $J=1,2,4,10,20,35$.

For reasons which will soon become clear we write this Hamiltonian as $H=(J-1) I+H_{0}+H_{1}(J)$, where $I$ is the unit matrix, $H_{0}$ is given by

and

$$
H_{0}=\left(\begin{array}{ccccc}
0 & g \sqrt{1} & & & \\
g \sqrt{1} & 1 & g \sqrt{2} & & \\
& g \sqrt{2} & 2 & g \sqrt{3} & \\
& & g \sqrt{3} & 3 & g \sqrt{4} \\
& & & g \sqrt{4} & 4
\end{array}\right),
$$




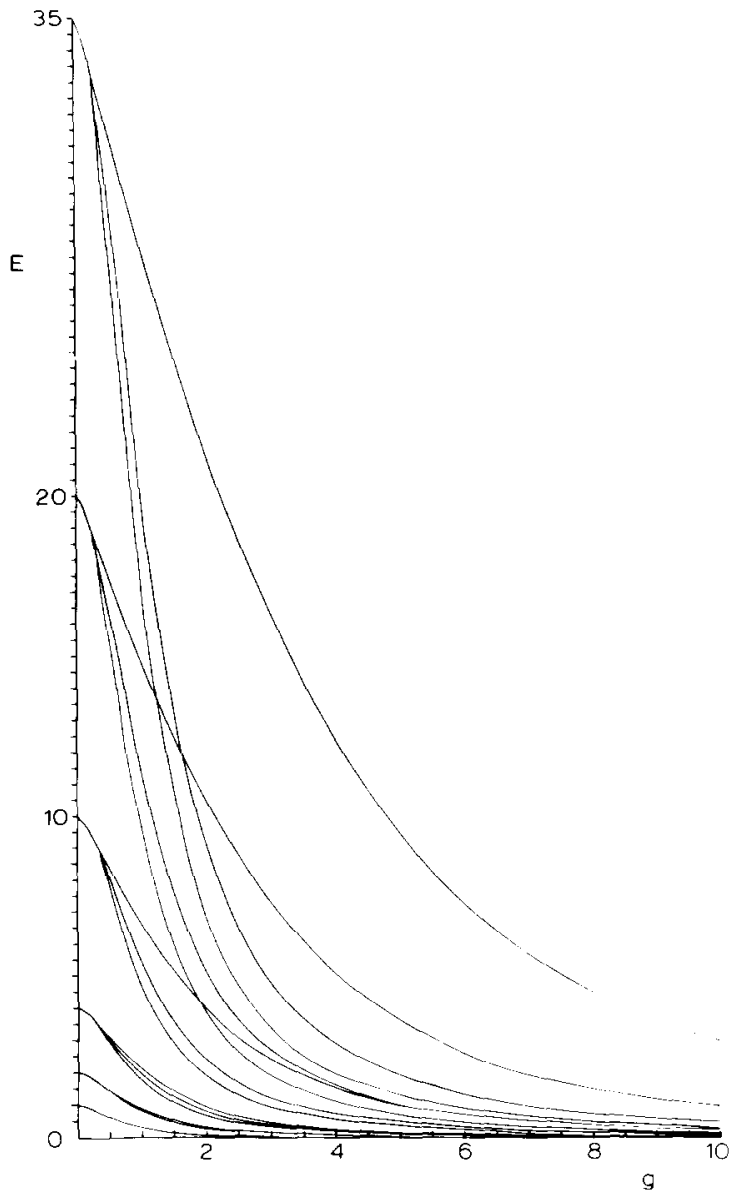

Fig. 5. Comparison of $[2,1]$ and $[3,2]$ Padé approximations with the exact value of $\epsilon+g^{2}+1$ for $J=1,2,4,10,20,35$. Of the three curves for each $J$ the upper one gives the exact value.

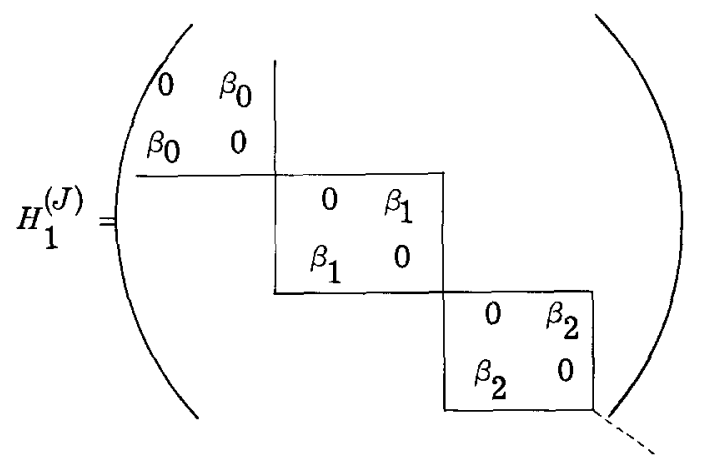

with 


$$
\beta_{n}=g \sqrt{2 N+2 J+1}-g \sqrt{2 n+1}, \quad n=0,1,2, \ldots .
$$

Introducing in an obvious way Boson-operators $a$ and $a^{*}$ (which have nothing to do with the original operators for the pion field) it is seen immediately that $H_{0}$ can be written as

$$
H_{0}=a^{*} a+g\left(a+a^{*}\right) \text {. }
$$

Defining new Boson-operators

$$
C=a+g
$$

$H_{0}$ becomes

$$
H_{0}=c^{*} c-g^{2} \text {. }
$$

The eigenvalues of $H_{0}$ are therefore $l-g^{2}$, with $l=0,1,2, \ldots$. The corresponding eigenstates will be denoted by $|l\rangle$. Also the spectrum of $H_{1}(J)$ is known. The eigenvalues are given by $\pm \beta_{n}(n=0,1,2, \ldots)$. This means that the discrete spectrum is contained between the limits $-\beta_{0}=-g[\sqrt{2 J+1}-1]$ and $+\beta_{0}$. Since $\lim _{n \rightarrow \infty} \beta_{n}=0$, the origin is the only accumulation point and it follows that $H_{1}(J)$ is a compact operator. It is therefore well suited to be used as perturbation for small $g$. Since we know the lower and upper bounds of the spectrum of $H_{1}(J)$ a well known theorem says that for the ground state the energy $E^{(J)}=\epsilon+g^{2}+1$ is enclosed between the limits

$$
J-g[\sqrt{2 J+1}-1]<E^{(J)}<J+g[\sqrt{2 J+1}-1] .
$$

For large coupling constant this is, however, a very weak constraint. In this case, we nevertheless take $H_{1}(J)$ as the perturbation and we will show that also for large $g$ this part of the Hamiltonian has only a small effect (e.g. on the position of the energy levels).

The main effect of strong coupling is the energy shift $-g^{2}$ of eq. (5.6), which we have incorporated in our unperturbed Hamiltonian. The reason why, even for large $g, H_{1}(J)$ has only small effects has to do with the following. Originally the Hilbert space was spanned by the eigenstates $|n\rangle$ of the operator $a^{*} a$. For the basic states of the same Hilbert space we now take the eigenstates $|l\rangle$ of $H_{0}$. The scalar product is found to be

$$
\langle n \mid l\rangle=\frac{(-1)^{n} g^{n+l} e^{-\frac{1}{2} g^{2}}}{\sqrt{n ! l !}} P_{(n, l)}\left(\frac{1}{g^{2}}\right),
$$

with

$$
P_{(n, l)}(x)=\sum_{t=0}^{M}(-1)^{t} t:\left(\begin{array}{l}
n \\
t
\end{array}\right)\left(\begin{array}{l}
l \\
t
\end{array}\right) x^{t}, \quad M=\operatorname{Min}(n, l) .
$$

For $g \rightarrow \infty$ all scalar products $\langle n \mid l\rangle$ tend to zero. This is similar to an effect, first pointed out by Van Hove [4], where the Hilbert space spanned by the dressed particles is orthogonal to the one spanned by the bare particles. From the fact that in the $|n\rangle$ representation the non-vanishing matrix elements of $H_{1}(J)$ tend to infinity we cannot conclude that the same happens in the $|l\rangle$ representation. In fact we probably have 


$$
\lim _{g \rightarrow \infty}\left\langle l_{1}\left|H_{1}^{(J)}\right| l_{2}\right\rangle=-J \delta l_{1} l_{2}
$$

The explicit proof has been given only for a few values of $l_{1}$ and $l_{2}$. We illustrate the method of proof for $l_{1}=l_{2}=0$.

Using eq. (5.7) one easily shows that

$$
\left\langle 0\left|H_{1}^{(J)}\right| 0\right\rangle=-2 e^{-g^{2}} \sum_{n=0}^{\infty} \frac{g^{4 n+2}}{(2 n+1) !} \gamma_{n}^{(J)},
$$

with

$$
\gamma_{n}^{(J)}=\sqrt{2 n+1}\{\sqrt{2 n+2 J+1}-\sqrt{2 n+1}\}
$$

If we expand

$$
\gamma_{n}^{(J)}=J-\frac{J^{2}}{2(2 n+2)}+\left(\frac{1}{n^{2}}\right),
$$

and substitute into eq. (5.9) we obtain

$$
\begin{aligned}
\left\langle 0\left|H_{1}^{(J)}\right| 0\right\rangle & =-2 J e^{-g^{2}} \sinh g^{2}+J^{2} \frac{e^{-g^{2}}}{g^{2}}\left(\cosh g^{2}-1\right)+\ldots \\
& =-J+\frac{J^{2}}{2 g^{2}}+\ldots,
\end{aligned}
$$

where the dots indicate terms of order $1 / g^{4}$ and of the form $e^{-2 g^{2}}$. From this we see that an expansion of $\left\langle l_{1}\left|H_{1}(J)\right| l_{2}\right\rangle$ in powers of $g^{-2}$ can at most be asymptotic and that $g=\infty$ is an essential singularity. A rather tedious calculation of the above type for a number of matrix elements gave the following results

$$
\begin{aligned}
& \left\langle 0\left|H_{1}^{(J)}\right| 0\right\rangle=-J+\frac{J^{2}}{2 g^{2}}-\frac{\left(J^{3}-J^{2}\right)}{2 g^{4}}+\ldots, \\
& \left\langle 0\left|H_{1}^{(J)}\right| 1\right\rangle=-\frac{J}{2 g}+\frac{3 J^{2}}{4 g^{3}}+\ldots, \\
& \left\langle 1\left|H_{1}^{(J)}\right| 1\right\rangle=-J+\frac{J^{2}}{2 g^{2}}+\ldots, \\
& \left\langle 0\left|H_{1}^{(J)}\right| 2\right\rangle=-\frac{J}{g^{2} \sqrt{2}}+\ldots, \\
& \left\langle 1\left|H_{1}^{(J)}\right| 2\right\rangle=-\frac{J}{g \sqrt{2}}+\ldots, \\
& \left\langle 2\left|H_{1}^{(J)}\right| 2\right\rangle=-J+\frac{J^{2}}{2 g^{2}}+\ldots .
\end{aligned}
$$


Assuming that asymptotically the matrix elements $\left\langle l_{1}\left|H_{1}{ }^{(J)}\right| l_{2}\right\rangle$ can be represented by a power series in $g^{-2}$ and that the leading term is of order $g-\left|l_{1}-l_{2}\right|$, we can also expand the components of the vector $X$ for the ground state

$$
X_{0}=1, \quad X_{1}=\frac{y_{1}}{g}+\frac{y_{2}}{g^{3}}+\ldots, \quad X_{2}=\frac{z_{1}}{g^{2}}+\frac{z_{2}}{g^{4}}+\ldots, \ldots .
$$

Writing the ground state energy as

$$
\epsilon=-g^{2}+\epsilon^{(0)}+\frac{\epsilon^{(1)}}{g^{2}}+\frac{\epsilon^{(2)}}{g^{4}}+\ldots,
$$

these expansions can be substituted into the Schrödinger equation. In this way we obtain

$$
\epsilon=-g^{2}-1+\frac{J^{2}}{4 g^{2}}+\frac{J^{2}}{4 g^{4}}+\ldots .
$$

In fig. 6 the functions (1): $J^{2} / 4 g^{2}$ and (2): $\left(J^{2} / 4 g^{2}\right)+\left(J^{2} / 4 g^{4}\right)$ are compared with the exact values of $E=\epsilon+g^{2}+1$. For $g^{2} \gtrsim J$ the agreement is very good.

Combining the results for weak coupling and for strong coupling we have calculated a "two-point" [4,3] Padé approximation to the quantity $\epsilon+g^{2}+1$, i.e.

$$
[4,3]\left(g^{2}\right)=\frac{A_{0}+A_{1} g^{2}+A_{2} g^{4}+A_{3} g^{6}}{1+B_{1} g^{2}+B_{2} g^{4}+B_{3} g^{6}+B_{4} g^{8}} .
$$

The asymptotic behaviour (5.11) gives two relations between the $A$ and $B$ coefficients. The power series (4.3) provides six relations, so that all coefficients in eq. (5.12) could be determined. For a number of $J$-values we have compared eq. (5.12) with the results of the exact numerical calculation. From fig. 7 it is seen that as long as $J$ is not too large the approximation is good for all values of the coupling constant. For large $J$, however, the approximation can develop spurious singularities. These have not been investigated systematically.

\section{DISCUSSION}

Using one of the simplest SU(2)-invariant theories for the interaction between pions and nucleons we showed that it is possible to treat the strong coupling limit in a more systematic way than has hitherto been done. As a result an extra term in the expansion of the ground state energy in inverse powers of the coupling constant could be determined. Another advantage is that because of the simplicity of the eigenvalue problem (2.6) it was feasible to perform a very accurate numerical calculation of the energy spectrum for a large range of the coupling constant and the isotopic spin. This allowed us to compare the exact results with a number of methods which are supposed to be good for small $g$, large $g$ or large $J$ and with a few Padé approximants. 


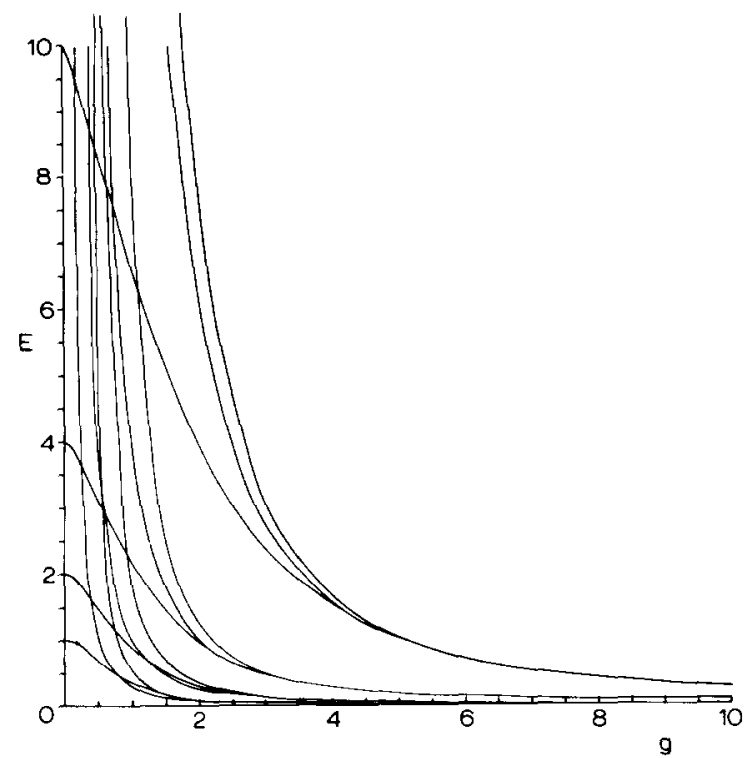

Fig. 6. Comparison of the functions (1) and (2) [see eq. (5.11)] with the exact value of $\epsilon+g^{2}+1$.

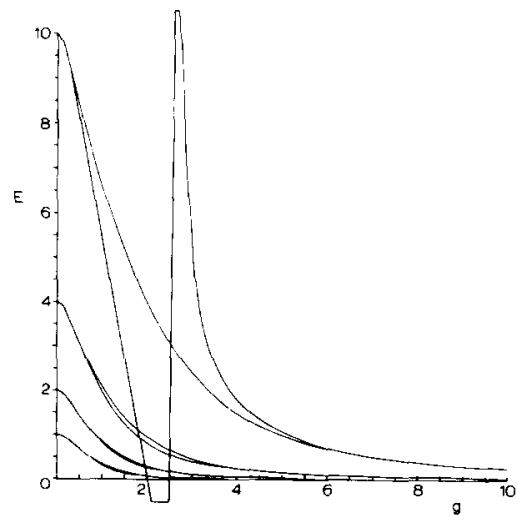

Fig. 7. Comparison of the two-point [4,3] Pade approximation with the exact value of $\epsilon+g^{2}+1$ for $J=1,2,4,10$. 


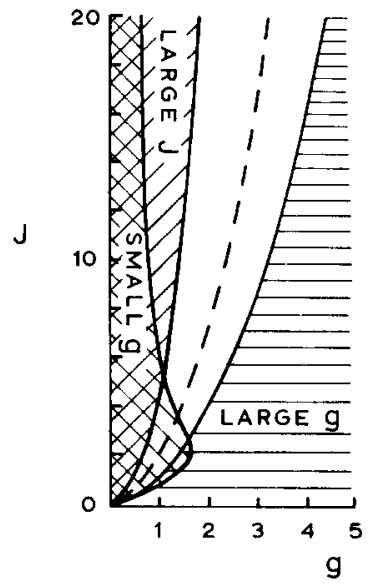

Fig. 8. Map of the $10 \%$ boundaries. Above the dotted line excited states will play a role.

For the different approximations we have determined the region in the $J-g$ plane in which the deviation from the exact result was less than $10 \%$. The boundaries of these regions are shown in fig. 8. An invasion of the terra incognito of this map seems to be possible from three sides. (i) From the "small $-g$ " region by calculating higher Padé approximants; (ii) From the "large $-g$ " region by calculating $g^{-6}$ terms; (iii) From the "large- $J$ " region by trying to find corrections to eq. (4.6).

For each $J$ we have only considered the ground state. Excited states could, however, be treated in the same way. Both for $g \rightarrow 0$ and for $g \rightarrow \infty$ the first excited state (for a given $J$ ) will be higher than the ground state by an amount of about one (pion mass). This shows that the formula $J^{2} / 4 g^{2}$ for the determination of the order of the different isospin states can be used only for values of $J$ such that $J \lesssim 2 g^{2}$. The boundary of the corresponding region in fig. 8 is indicated by a dotted line. For higher $J$ values the excited states must be taken into account.

The author is indebted to Dr.J. Tjon for stimulating discussions. He thanks Mr.J. Renes for performing the numerical calculations.

\section{APPENDIX}

In this appendix we derive the formulae which must be used in order to write the left hand side of eq. (2.3) as a sum of tensor operators of the form $T_{k}^{q} G^{* n}|0\rangle$. Since

$$
D=a_{j}^{*} a_{j}, \quad \text { (summation convention), }
$$

is the number operator and $G^{*}|0\rangle=a_{j}^{*} a_{j}^{*}|0\rangle$ contains two particles we have 


$$
D T_{k}^{q} G^{* n}|0\rangle=(2 n+k) T_{k}^{q} G^{* n}|0\rangle
$$

In what follows we will need the isospin of the meson field, i.e.

$$
I_{j}=-i \epsilon_{j k l} a_{k}^{*} a_{l}
$$

and the operators $a_{ \pm}^{*}=\frac{1}{2} \sqrt{2}\left(a_{1}^{*} \pm i a_{2}^{*}\right), a_{0}^{*}=a_{3}^{*}$. Using $I_{-}=\sqrt{2}\left\{a_{-}^{*} a_{0}-a_{0}^{*} a_{+}\right\}$ and the definition of $T_{J} J$ (sect. 2) it can be shown that from $\left[I_{-}, T_{J} J^{J}=\sqrt{2 J} T_{J}^{J-1}\right.$ follows:

$$
a_{0}^{*} T_{J-1}^{J-1} G^{* n}|0\rangle=-T_{J}^{J-1} G^{* n}|0\rangle
$$

Application of $I_{-}$and using $\left[I_{-}, a_{+}^{*}\right]=-\sqrt{2} a_{0}^{*}$ gives

$$
a_{+}^{*} T_{J}^{J-1} G^{* n}|0\rangle=\sqrt{J} T_{J+1}^{J} G^{* n}|0\rangle \text {. }
$$

From eq. (A.2) (replacing $J$ by $J+1$ ) follows

$$
I_{-} a_{0}^{*} T_{J}^{J} G^{* n}|0\rangle=-\sqrt{2(2 J+1)} T_{J+1}^{J-1} G^{* n}|0\rangle \text {. }
$$

Combining this with $\left[I_{-}, a_{0}^{*}\right]=\sqrt{2} a_{-}^{*}$ and $a_{-}^{*} a_{+}^{*}=\frac{1}{2}\left(G^{*}-a_{0}^{*} a_{0}^{*}\right)$ gives

$$
a_{0}^{*} T_{J}^{J-1} G^{* n}|0\rangle=-\frac{1}{2 J+1} T_{J-1}^{J-1} G^{* n+1}|0\rangle-2 \sqrt{\frac{J}{2 J+1}} T_{J+1}^{J-1} G^{* n}|0\rangle \text {. }
$$

Using $a_{j} G^{* n}|0\rangle=2 n a_{j}^{*} G^{* n-1}|0\rangle$, one derives in a similar way

$$
\begin{gathered}
a_{0} T_{J-1}^{J-1} G^{* n}|0\rangle=-2 n T_{J}^{J-1} G^{* n-1}|0\rangle, \\
a_{-}^{*} T_{J}^{J} G^{* n}|0\rangle=\frac{\sqrt{J}}{2 J+1} T_{J-1}^{J-1} G^{* n+1}|0\rangle-\frac{1}{\sqrt{2 J+1}} T_{J+1}^{J-1} G^{* n}|0\rangle,
\end{gathered}
$$

and

$$
a_{+} T_{J}^{J} G^{* n}|0\rangle=\sqrt{J} T_{J-1}^{J-1} G^{* n}|0\rangle+2 n a_{-}^{*} T_{J}^{J} G^{* n-1}|0\rangle \text {. }
$$

This last equation together with eq. (A.6) gives

$a_{+} T_{J}^{J} G^{* n}|0\rangle=\frac{\sqrt{J}}{2 J+1}(2 n+2 J+1) T_{J-1}^{J-1} G^{* n}|0\rangle-\frac{2 n}{\sqrt{2 J+1}} T_{J+1}^{J-1} G^{* n-1}|0\rangle$.

Applying $I_{-}$to eq. (A.5) (with $J \rightarrow J+1$ ) gives

$a_{0} T_{J}^{J-1} G^{* n}|0\rangle=-\frac{(2 n+2 J+1)}{2 J+1} T_{J-1}^{J-1} G^{* n}|0\rangle-4 n \sqrt{\frac{J}{2 J+1}} T_{J+1}^{J-1} G^{* n-1}|0\rangle \cdot$ (A.8)

It is now straightforward, using eqs. (A.1)-(A.8), to perform the reduction of eq. (2.3) of the main text and to derive eq. (2.5).

For the normalization we must calculate 


$$
Z_{n}(J) \equiv\left\langle 0\left|G^{n} a_{+}^{J} a_{+}^{* J}{ }^{* n}\right| 0\right\rangle
$$

Using the auxiliary formulae

$$
G^{* n}|0\rangle=2 n(2 n+1) G^{* n-1}|0\rangle,
$$

and

$$
\left[G, a_{+}^{* J}\right]=2 \int a_{+}^{* J-1} a_{-},
$$

we derive

$$
\begin{aligned}
Z_{n}(J)= & \left\langle 0\left|G^{n-1} a_{+}^{J}\left\{2 J a_{+}^{* J-1} a_{-}+a_{+}^{* J} G\right\} G^{* n}\right| 0\right\rangle \\
& =4 n J\left\langle 0\left|G^{n-1} a_{+}^{J} a_{+}^{* J} G^{* n-1}\right| 0\right\rangle+2 n(2 n+1)\left\langle 0\left|G^{n-1} a_{+}^{J} a_{+}^{* J} G^{* n-1}\right| 0\right\rangle,
\end{aligned}
$$

or

$$
Z_{n}(J)=2 n(2 n+2 J+1) Z_{n-1}(J) .
$$

Since $Z_{0}(J)=\left\langle 0\left|a_{+}^{J} a_{+}^{* J}\right| 0\right\rangle=J$ : we obtain

$$
Z_{n}(J)=\left\langle 0\left|G^{n} a_{+}^{J} a_{+}^{* J} G^{*}\right| 0\right\rangle=\frac{(J !)^{2}}{(2 J+1) !} \frac{n !(2 n+2 J+1) !}{(n+J) !} .
$$

\section{REFERENCES}

[1] T. Cook, C. J. Goebel and B. Sakita, Phys. Rev. Letters 15 (1965) 35;

C. J. Goebel, Proc. of the 1965 Midwest Conf. on theoretical physics (Ohio State University, Columbus, Ohio) p.63;

C. J. Goebel, Phys. Rev. Letters 16 (1966) 1130;

C. Dullemond, Ann. of Phys. 33 (1965) 214;

C. Dullemond and F. J. M. Von der Linden, Ann. of Phys. 41 (1967) 372;

M. Bedná and J. Tolar, Revised version of Dubna preprint E2-3044 (1966).

[2] G. Wentzel, Helv. Phys. Acta 13 (1940) 269; 14 (1941) 633;

W. Pauli and S. M. Dancoff, Phys. Rev. 62 (1942) 85;

R. Serber and S. M. Dancoff, Phys. Rev. 63 (1943) 143;

A. Pais and R. Serber, Phys. Rev. 105 (1957) 1636;

H. Nickle and R. Serber, Phys. Rev. 119 (1960) 449;

H. Jahn, Z. Phys. 156 (1959) 633;

H. Jahn, Fortschr. Phys. 7 (1959) 452.

[3] J.D. Swalen and L. Pierce, J. Math. Phys. 2 (1961) 736;

L. Pierce, J. Math. Phys. 2 (1961) 740.

[4] L. Van Hove, Physica 18 (1952) 145.

[5] E. M. Henley and W. Thirring, Elementary quantum field theory (McGraw-Hill Book Company, Inc., New York, 1962) pp. 188-192.

[6] E. Henley and T. D. Lee, Phys. Rev. 101 (1956) 1536, eq. (57). 